\title{
ADDRESSING THE CRISIS IN FUNDAMENTAL PHYSICS
}

\author{
CHRISTOPHER W. STUBBS \\ Department of Physics \\ $\&$ \\ Department of Astronomy \\ Harvard University \\ 17 Oxford Street, Cambridge MA USA 02138 \\ cstubbs@fas.harvard.edu
}

Received __; accepted _ 


\section{Introduction: The "Standard Models" of Particle Physics and Cosmology}

Over the course of one human lifetime we have developed two powerful "standard models". One pertains to particle physics while the other is cosmological. On the particle physics front we know of 3 families of quarks and leptons, which interact via exchange bosons. This picture is known to be incomplete: We don't know how to fit gravity into this scenario, and the dark matter continues to elude us.

On the cosmological side, driven in no small measure by the recent WMAP

measurements of the structure in the cosmic microwave background (1) we now assert that:

- Our geometrically flat Universe started in a hot big bang 13.7 Billion years ago.

- The matter component of the Universe is dominated by dark matter, which is most likely outside the scope of the particles that make up the standard model of particle physics.

- Luminous matter comprises only a few percent of the total mass of the Universe.

- The evolution of the Universe is increasingly dominated by the phenomenology of the vacuum.

This consensus cosmology is, however, ludicrous. The assertion that two regions of the vacuum experience a mutually repulsive gravitational interaction is, well, repulsive. With apologies to Kirk, Scotty and especially Mr. Spock, it's like living in a bad episode of Star Trek. It is worth asking, therefore, why this preposterous consensus has emerged, and on the basis of what experimental data.

The ingredients of the Universe are most conveniently expressed in terms of a cosmic sum rule, where

$$
\Omega_{k}+\Omega_{\Lambda}+\Omega_{m}=1
$$


with $\Omega_{k}$ representing the contribution from any underlying curvature in the underlying geometry of the Universe, $\Omega_{\Lambda}$ reflects the contribution of the Dark Energy component, and $\Omega_{m}$ accounts for the matter density of the Universe (in units of the critical density).

\section{The Observational Evidence for Dark Energy}

The first claims for an accelerating expansion of the Universe were made in the late 1990's by two teams (2), (3) that used type Ia supernovae to probe the history of cosmic expansion. Supernovae at redshifts around 0.7 were seen to have luminosities that were about $20 \%$ fainter than expected in a well-behaved Universe.

As with nearly all results in observational cosmology, the supernova measurements have the potential for unappreciated systematic error, and the type Ia Hubble diagram alone is (in my view) insufficient to compel us to believe in the Dark Energy, with $\Omega_{\Lambda} \sim 0.7$.

The sobering fact is that essentially all subsequent cosmological data sets drive us to the conclusion that the Universe has recently entered a time in which the scale factor is growing exponentially. The supernova data, measurements of primordial element abundances, determinations of the overall mass density of the Universe, and the structure seen in the microwave background all support this conclusion (4).

Cognizant of the danger of making a list that appears to be comprehensive but may not be, the possibilities for the underlying physics include:

1. A classical cosmological constant, residing in the gravitational physics sector,

2. Vacuum energy effects, arising from quantum mechanical fluctuations, and

3. A modification of gravity that is manifested on the cosmological scale. 
The good news aspect of this situation is that we have clear evidence of new physics. The bad news is that we presently have no idea what it means.

\subsection{Dark Energy Constitutes a Crisis in Fundamental Physics}

In my opinion the Dark Energy situation constitutes a crisis in fundamental physics that is every bit as profound as that which preceded the advent of quantum mechanics.

One might wonder if perhaps we are somehow being misled by the data, or that the data are being misinterpreted. The convergence of results from such a wide array of

measurement techniques argues against this, but we should nevertheless retain our sense of scientific scepticism. Under such circumstances we might turn perhaps to theory for some guidance...

\section{The Present Theoretical Situation}

From the our current perspective there are only two natural theoretical values for $\Omega_{\Lambda}$, the apparent contribution of the vacuum energy in units of the critical density. The first of these natural values comes from integrating the effect of all known quantum mechanical fluctuations, up to the Planck mass. The resulting value of $\Omega_{\Lambda}=10^{120}$ is so preposterous that it's a non-starter. The other natural value presupposes that some cancellation mechanism steps in and performs a precise cancellation, leaving $\Omega_{\Lambda}=0$ (to 120 significant 
figures!).

In the context of an observed value of $\Omega_{\Lambda} \sim 0.7$, this presents us with a problem. Some scholars have advocated that anthropic selection effects provide an explanation for this rather unexpected value, while others retain hope that a future theory will provide some natural explanation.

Today, in the absence of a clear theoretical framework with relevant predictive power, the Dark Energy problem is best characterized as a data and discovery driven endeavor, spiced with interesting theoretical speculation.

\section{The Present Observational Situation}

At the present time observational cosmologists are concentrating on measuring the equation of state parameter of the dark energy, $w=P / \rho$, the value of which may help us distinguish between the different possible mechanisms that might underlie the Dark Energy. Current and upcoming cosmological observations exploit gravitational lensing, cluster abundances, baryon acoustic oscillations, and the supernova Hubble diagram to improve our understanding of the Dark Energy. These all have the merit of being undertaken in a regime where the signal is non-zero, but have the shared disadvantage of being susceptible to the various sources of systematic error that afflict astronomical observations. The desire to discriminate between different Dark Energy models will push these techniques to their limits.

At the time of this writing (mid 2006) we are starting to see preliminary determinations of $w=P / \rho$, the equation of state parameter of the Dark Energy, and its evolution over cosmic time. One such example is from the ESSENCE supernova survey, in which I participate. When the supernova data are combined with constraints from large scale 
structure(5), the data favor $w=-1$. Similar results have been reported (ㅁ) by the CFHT Legacy Survey team. Unfortunately this value that has the least power to discriminate the origin of the physics that is driving the accelerating expansion.

\subsection{It Could Get Grim}

Looking ahead, there is a very real possibility that cosmological measurements will show that $w=-1$ at all accessible redshifts. This would be a grim circumstance, and our attempt to discover the nature of the dark energy through cosmological observations would completely stall.

Although science has very recently made huge strides in understanding the nature of the reality we inhabit, there are certainly ample examples in the past of scientific fields going through long periods of stagnation. I fear that if we arrive at $w=-1$, we may be facing such a fate unless we find another piece in this jigsaw puzzle.

\section{Precision Fundamental Physics Experiments and the Dark Energy Problem.}

Many of the speculative ideas that have been put forward to explain the dark energy produce observable effects in other domains. We can therefore hope that a vigorous program of precision measurements and tests of fundamental physics might produce another anomalous result, which when integrated with the cosmological acceleration will lead us to a deeper understanding. Of course we don't know where this anomaly might arise, and this motivates our undertaking a broad array of experiments, including

- Testing our understanding of gravity on all scales, including the inverse square law, 
the equivalence principle, the strong gravity regime, and gravitomagnetism.

- Direct tests of the fabric of spacetime, including geometrical flatness tests, precision clock experiments, time delay measurements, etc.

- Tests of fundamental symmetries and Lorentz invariance,

- Probing the nature of vacuum fluctuations and Casimir forces.

Many of these projects have a long history in the precision measurement and fundamental physics arenas. In my opinion the broader community's appreciation of their importance is likely to grow as we collectively turn to face the unexpected crisis posed by the Dark Energy.

\section{The Role of Space-based Projects}

We should distinguish at the outset between space-based observations and experiments. On the observational side, we exploit the low IR background, diffraction-limited imaging, and predictable absence of weather to collect photons from distant sources. We then use these observations to infer the properties of the Universe we inhabit.

This is to be contrasted with space-based experiments, where the absence of seismic perturbations, the microgravity environment, and access to various solar system gravitational potentials are combined with precision apparatus to pose well-defined experimental questions of nature.

The ability to carry out precision measurements, in which we can explicitly test the system's susceptibility to potential sources of systematic error, gives experiments a special role to play in probing for a deeper understanding of Dark Energy. 
Our challenge is to identify those instances where we can realize large gains by combining the tools and techniques from the precision metrology and fundamental measurement communities with the special environmental aspects of space. Once these opportunities are identified we face our next daunting challenge, namely prioritizing the different options when we (for now, at least) have no idea where the signature for new physics might next emerge. As articulated by Dr. Marburger in his opening remarks at this meeting, far better that we scientists make that assessment than leaving it to lobbyists and legislators.

\section{An Example- Laser Ranging in the Solar System.}

This meeting has numerous interesting presentations on existing and proposed fundamental physics projects, many of which might exhibit the next piece of evidence for new physics. Let me pick one illustrative example and indicate how it links to the Dark Energy problem.

Smith et al. have recently demonstrated(7) the successful exchange of laser communication signals with a spacecraft a distance 24 million $\mathrm{km}$ from Earth. See also the talk by Degnan in these proceedings. This gives reality to the notion of "piggybacking" a precision laser ranging capability on laser communication links. If NASA holds to its current goals of missions to the moon and Mars, we could imagine adding a fundamental physics aspect to these flight opportunities. (See the paper by Merkowitz in this volume, and references therein).

The scientific merit of an aggressive and coordinated solar system ranging campaign has been outlined(8) by Nordtvedt, using the various gravitational interactions between the bodies in the solar system. Performing a global fit to ranging data between the Earth, 
the moon, and the other planets will allow us to perform a comprehensive test of the basic foundations of gravity.

Successfully undertaking projects of this sort will require a coordination of efforts individuals and teams drawn from diverse communities, including:

- Precision measurement and metrology,

- Atomic-Molecular-Optical physics,

- Astronomy,

- Gravitational theory and numerical analysis,

- Particle physics,

- Precision engineering, and

- Astronautics and spacecraft engineering.

This meeting is a great opportunity for us to renew old partnerships, and to build new ones.

\section{An Exhortation}

Let me then end with an exhortation. We are in the midst of a profound crisis in fundamental physics. On the theoretical side, we know that our two primary triumphs, namely general relativity and quantum mechanics, do not work well together. On the observational side we have the challenge of the increasing evidence for the accelerating expansion of the Universe. I think there is a very real possibility that the observational 
cosmologists will, in the decades ahead, present us with increasingly precise measurements of $w=-1$, with no evidence for evolution over cosmic time.

This will require that we push the frontiers of fundamental physics in order to search for the next piece of the Dark Energy puzzle. In my view the task of this meeting is to identify and refine the concepts that stand the best chance of capitalizing on space flight opportunities to address this crisis in fundamental physics.

\section{Acknowledgments}

I would like to thank the organizers for putting together such a stimulating meeting. In addition I would like to thank both Slava Turyshev and Thomas Murphy for their invitation, and for their generous help, encouragement, and valuable comments while I was preparing this talk. I am also grateful to Harvard University, to the Department of Energy (through their grant to Harvard's Laboratory for Particle Physics and Cosmology) and to the National Science Foundation (under grant AST-0507475) for supporting my own work on the Dark Energy problem. 


\section{REFERENCES}

D. Spergel and the WMAP team, astro-ph/0603449 (2006).

A. Riess et al, $A J$ 116, 1009 (1998).

S. Perlmutter et al ApJ 517565 (1999).

W. Freedman, and M. Turner, Rev Mod Phys 75, 1433 (2003)

G. Miknaitis et al, BAAS 20715203 (2005).

P. Astier et al, $A \mathscr{E} A$ 447, 31 (2006).

Smith et al, Science 311, 53 (2006)

K. Nordtvedt, it Phys Rev D 61, 122001 (2000). 